\title{
Incorporation of Mechanical Noise in the SPICE Model of a Piezoelectric Transducer
}

\author{
Jonny Johansson ${ }^{1}$, Johan Borg \\ EISLAB, Dept. of Computer Science and Electrical Engineering, \\ Luleå University of Technology, SE-971 87 Luleå, Sweden. \\ ${ }^{1}$ Email: jonny.johansson@1tu.se
}

\begin{abstract}
SPICE models of a piezoelectric device and the ultrasound propagation medium can be used in a simulator intended for electronic circuits and IC design to make efficient system level optimizations. This paper presents the inclusion of mechanical noise in the SPICE model of an ultrasound system. The modeling of the noise is based on the mechanical thermal noise which is equivalent to electronic Johnson (thermal) noise. For a system with a high-Q piezoelectric device designed into a medium- $\mathrm{Q}$ transducer the main energy loss, and thus also the main noise contribution, will occur in backing and sound propagation media. Thus, the modeling of the mechanical noise is performed by including electrical noise generation in the resistors that model these media in the electrical equivalent circuit. The resulting output voltage noise follows theoretical derivations of transducer noise as published by Farlow and Hayward. Simulations of a $4 \mathrm{MHz}$ Pz27 piezoelectric disc with a diameter of $8 \mathrm{~mm}$ give a peak spectral noise density over $1 \mathrm{nV} / \sqrt{\mathrm{Hz}}$, which is comparable to that achievable with low-noise preamplifiers.
\end{abstract}

Keywords: PSpice modeling, noise density, piezoceramic, ultrasound

\section{A Introduction}

Simulation tools originally intended for electronic circuits (e.g. SPICE, PSpice) have become established in the design of ultrasound systems through the introduction of equivalent circuits for piezoelectric transducers. The models map force and particle velocity in the mechanical domain onto voltage and current in the electrical domain. The core of the transducer model is a transmission line, which simulates the mechanical resonator. The models have evolved over time. Initially developed in [1], [2], [3], they were further improved by the introduction of controlled sources in order to avoid transformers and negative capacitances [4]. Mechanical and viscoelastic loss have been introduced to model low Q transducers [5]. The effect of material properties on viscoelastic loss was further investigated in [6] to improve the accuracy of the models regarding loss and wave speed. Recently, the influence of diffraction and parasitic components have been included in the models, which makes it possible to achieve correct simulation of absolute amplitude levels in a pulse echo system [7].

One targeted usage area for theses models is to optimize an electronic-acoustic system with regard to for example dynamic range (DR), power consumption, and signal-tonoise ratio (SNR). In most cases these parameters are interconnected such that an increased design requirement in dynamic range or SNR will require an increase in power consumption. Optimization of both dynamic range and SNR will require knowledge of the system noise level. In many cases, this is set by the electronics, for which also noise modeling is well established and accurate [8]. However, cases do exist where the influence of the mechanical noise generated in the piezoelectric transducer reaches levels in parity with the electronic noise in a low noise amplifier, and thus has to be taken into account. A number of publications has previously touched on this subject, both expressing noise as minimum detectable force [9] and including the noise effects in the KLM equivalent model [10]

In this paper it is shown that simulation of mechanical noise is possible using a PSpice implementation of the Leach model of a piezoelectric transducer. The intention is to allow straightforward use of the model in situations were the target is low-noise electronics design, and the mechanical noise needs to be incorporated to avoid sub-optimized solutions. The following part of this paper introduces the equivalent circuit in more detail, and section three presents the method used to map mechanical noise onto electrical properties. Thereafter, simulation results and discussion are presented.

\section{B The Leach model}

The electrical model of a piezoelectric device as derived by Leach [4] is shown in Fig. 1. Here, $I_{p z}$ and $V_{p z}$ are current and voltage on the electrical side of the transducer. The force acting on the surface of the transducer is denoted $F$ and the particle velocity in the material $U_{p z}$. The parameter $c^{D}$ is the open circuit elastic constant, and $h$ is defined as

$$
h=\frac{e}{\varepsilon^{S}} .
$$

The equations that describe the one-dimensional wave propagation in a piezoelectric crystal can in the Laplace domain be written

$$
\frac{d}{d x}\left[F-\frac{h}{s} I_{p z}\right]=-\rho A s U_{p z}
$$

and

$$
\frac{d}{d x} U_{p z}=-\frac{s}{A c^{D}}\left[F-\frac{h}{s} I_{p z}\right]
$$




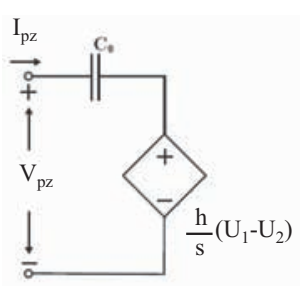

a) Electrical Part

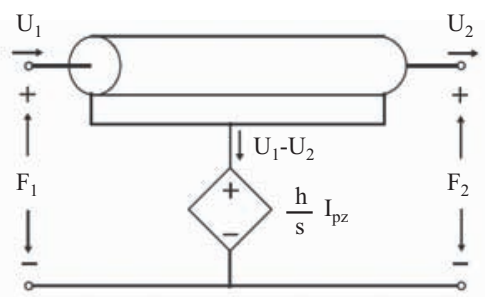

b) Mechanical Part
Fig. 1. Leach' equivalent circuit of a piezoelectric device.

where $\mathrm{A}$ is the area of the transducer.

As shown in Fig. 1, an electrical transmission line is used to model the resonant mechanical part of the transducer. Moving back to this electrical domain, the wave propagation in a lossless transmission line can be described by the telegraphist's equations [11];

$$
\frac{d}{d x} V_{t r}=-L_{t r} s I_{t r}
$$

and

$$
\frac{d}{d x} I_{t r}=-C_{t r} s V_{t r}
$$

Here $I_{t r}$ is the current and $V_{t r}$ is the voltage in the transmission line. Further, $L_{t r}$ and $C_{t r}$ are the inductance and capacitance per unit length of the transmission line. Thus, the assignment of

$$
V_{t r}:=\left[F-\frac{h}{s} I_{p z}\right]
$$

and

$$
I_{t r}:=U_{p z}
$$

reveals the equivalence between the wave propagation in the transducer and the transmission line. The model as depicted in Fig. 1b can then be used to model the mechanics of the transducer with a transmission line that has the properties

$$
L_{t r}=\rho A
$$

and

$$
C_{t r}=\frac{1}{A c^{D}}
$$

With this, the velocity in the transmission should be set to

$$
v_{t r}=\frac{1}{\sqrt{L_{t r} C_{t r}}}=\sqrt{\frac{c^{D}}{\rho}}=v_{D},
$$

where $v_{D}$ is the mechanical wave velocity (i.e. speed of sound) in the piezoelectric transducer. Further, the characteristic impedance in the line should be set to

$$
Z_{0, t r}=\sqrt{\frac{L_{t r}}{C_{t r}}}=\rho A v_{t r}=A Z_{a}
$$

where $Z_{a}=\rho v_{t r}$ is the specific acoustic impedance of the transducer.

To complete the electrical side of the model, the resulting voltage $V_{p z}$ can be shown to be

$$
V_{p z}=\frac{h}{s}\left[U_{1}-U_{2}\right]+\frac{1}{C_{0} s} I_{p z} .
$$

Here, $U_{1}$ and $U_{2}$ are the velocities of the surfaces of the disc, and

$$
C_{0}=\frac{\varepsilon^{S} A}{l}
$$

is the static capacitance for the piezoelectric disc with the clamped permittivity $\varepsilon^{S}$ and thickness $l$. The result from equation 12 is included in the Leach model as depicted in Fig. 1a. Standard SPICE does not allow frequency dependent sources. The frequency dependence can instead be modelled using a different approach as seen in Fig. 2 and Fig. 4. The integration of $\left[U_{1}-U_{2}\right]$ in equation 12 is achieved by the use of a dependent current source together with $C_{0}$. In the mechanical part of the model a dependent current source is used together with an added capacitor $C_{i}$ of $1 \mathrm{~F}$ to achieve the integration of $I_{p z}$ for equation 6 . The resistor $R_{i}$ has been added to avoid a floating node.

A transmission line is also used when wave propagation is modelled in a mechanical medium. In this case there is no piezoelectric effect to model, and the assignment becomes

$$
V_{t r, m}:=F_{m}
$$

and

$$
I_{t r, m}:=U_{m},
$$

where $I_{t r, m}$ is the current and $V_{t r, m}$ is the voltage in the transmission line that models the medium. $F_{m}$ is the force acting on the medium and $U_{m}$ is the particle velocity on the surface of the medium.

\section{Noise}

In 1928, Johnson and Nyquist published two papers describing a noise effect in conductors which is evenly distributed over frequency, i.e. white noise [12], [13]. They showed that this noise is an effect of the resistance $R_{E}$ in the conductor as well as the absolute temperature $T$, and that it gives rise to randomly distributed voltage fluctuations as

$$
V_{n, R M S}=\sqrt{4 k_{B} R_{E} B},
$$

where $k_{B}$ is the Boltzman constant and $B$ is the bandwidth of the noise. This noise is generally simulated with high accuracy in simulators for electrical circuits, e.g. SPICE and Spectre [8]. When resistors are present in the electrical circuit they can be set to generate noise, and the ensuing noise simulation is thereafter performed in the frequency domain to get either integrated noise over a certain bandwidth, or noise as $\mathrm{V} / \sqrt{\mathrm{Hz}}$ plotted over frequency.

In 1951, Callen and Welton [14] extended the Nyquist relation and showed it to be valid not only in an electrical system, but also in any general linear dissipative mechanical system. One case was in 2001 published by Farlow and Hayward [9], where they show that the force fluctuations in a solid are linked to the real part $R_{M}$ of the mechanical impedance as

$$
F_{n, R M S}=\sqrt{4 k_{B} R_{M} B} .
$$

Although derived in the context of an air coupled ultrasound transducer, this relation can be shown to be generally valid.

From this it can be concluded that given the assignments in equations 14 , and 11 , we can directly use equation 16 


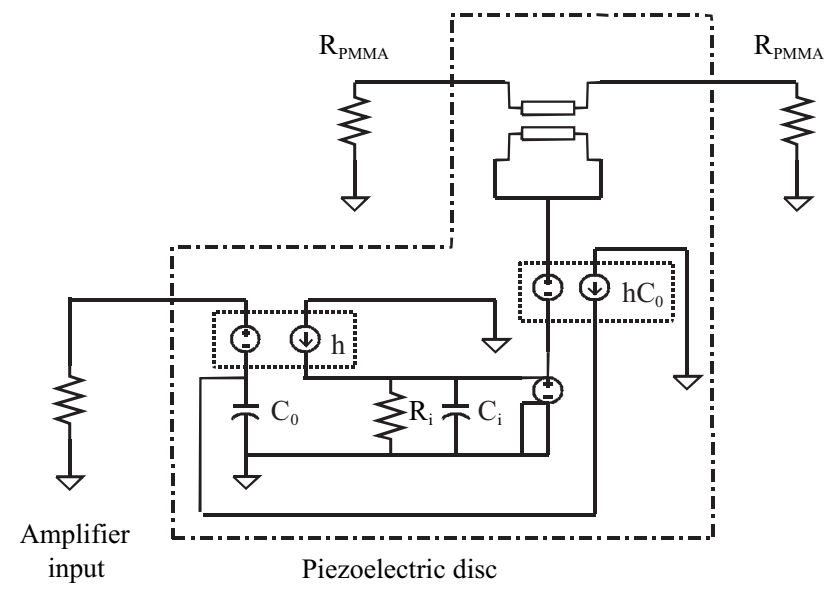

Fig. 2. Simulation setup with the piezoelectric disc loaded with infinitely thick PMMA on both front and rear.

in the Leach model to estimate the mechanical noise that arises as a result of equation 17. Although this is directly true for the passive parts of the system, i.e. the transmission media, it will also hold for the piezoelectric device if it is modelled as open circuit, as then $I_{p z}=0$ and the assignment in 6 reduces to

$$
V_{t r}:=F
$$

The noise considered in this paper is directly related to energy loss, whether electrical in a resistor or mechanical. Considering the piezoelectric disc itself, this is commonly low loss. For a piezoceramic Pz27 disc with a resonance frequency of $4 \mathrm{MHz}$, the Q-value of the unloaded disc is typically in the order of $70<Q_{p z}<80$. When the disc is used to build a transducer with matching and backing layers, it is often desired to reduce the Q-value for the transducer to achieve short broadband pulses. Most of the loss of energy in the system then occurs not in the transducer, but in the backing and in the medium to be investigated. Thus, the main mechanical noise is mainly contributed from those parts. In the simulation, this contribution can then be modelled by noise generation in the resistors that model these materials, as further described below.

\section{Simulations and discussion}

Consider an ultrasound system where the piezoelectric disc is glued between two infinite plates of plexiglas, PMMA. The Leach model of such a system is shown in Fig. 2. As the PMMA is infinite, it can here be modelled as a resistance with the value of the mechanical acoustic impedance of the PMMA, i.e.

$$
R_{P M M A}=\pi a^{2} \rho_{P M M A} c_{P M M A} .
$$

Here, the radius of the transducer is $a=4 \mathrm{~mm}$, the density of PMMA is $\rho_{P M M A}=1190 \mathrm{~kg} / \mathrm{m}^{3}$, and the speed of sound in PMMA is $c_{P M M A}=2775 \mathrm{~m} / \mathrm{s}$, leading to $R_{P M M A}=166 \Omega$. The characteristic impedance of the transmission line that models the piezoelectric disc is

$$
Z_{0 P Z}=\rho_{P Z} \pi a^{2} c_{P Z},
$$

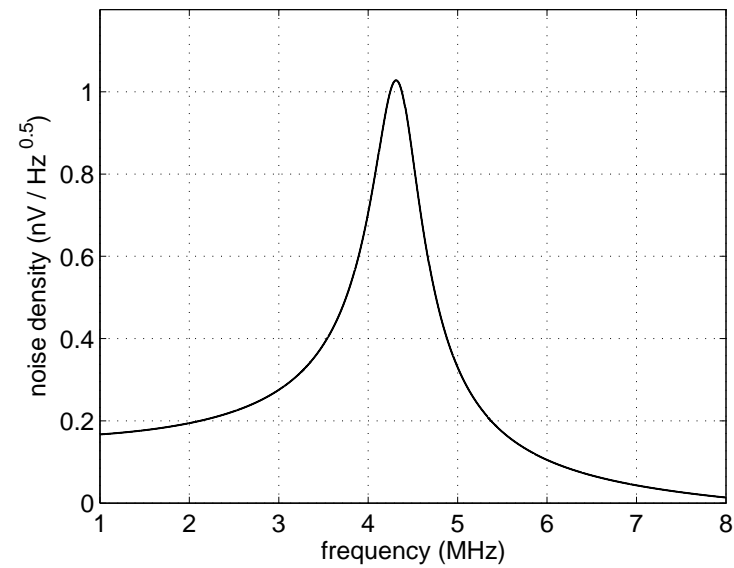

Fig. 3. Noise spectral density at transducer output when loaded with infinitely thick PMMA on both front and rear.

which with $c_{P Z}=4334 \mathrm{~m} / \mathrm{s}$ and $\rho_{P Z}=7700 \mathrm{~kg} / \mathrm{m}^{3}$ equates to $Z_{0 P Z}=1677 \Omega$. The Q-value for this setup that results forom the assumption of a lossless piezoelectric disc can be calculated as [15]

$$
Q=\left(2 Z_{0 P Z}\right) /\left(\pi R_{P M M A}\right)=6.43
$$

The simulation was run using the Cadence Spectre simulation engine. In the simulation, the only resistors set to generate noise were the two representing the PMMA. The resulting output noise at $R_{a m p}$ has been plotted over frequency in Fig. 3. To verify the correctness of this result, a comparison was made with noise plotted as derived for identical conditions by Farlow and Hayward [15]. The results from this comparison are identical. As can be seen in Fig. 3, the noise reaches levels exceeding $1 \mathrm{nV} / \sqrt{\mathrm{Hz}}$ around the center frequency of the transducer. This level is comparable to that achievable with low-noise preamplifiers, which underscores the relevance of the noise inclusion in the model.

The previous example is fairly straightforward to evaluate analytically as shown in [15]. The versatility of the PSpice modeling approach is however clear if a second example is considered. Here, as shown in Fig. 4, the piezoelectric disc is immersed in water. A pulse echo system is created by sending a pulse through $5 \mathrm{~mm}$ of water after which it is reflected at a PMMA reflector. For the simulation, it is once again assumed that the piezoelectric disc is lossless. Furthermore, as water is a low-loss medium, and the distance is short, also the loss occurring in the water are neglected. Thus, the only noise contribution to the mechanical side arise in the resistors $R_{\text {Backing }}$ and $R_{P M M A}$. The results from two simulation runs with different backing materials are shown in Fig. 5. The curve with a higher Q-value was run with PMMA as backing material. For the second run, the impedance of the backing material was increased a factor of five. The resulting curve shows clearly the effect on the noise shaping for the different backing materials. The increase in backing impedance lowers the Q-value, and consequently also lowers the peak noise spectral density with a factor of two. Both curves also show 


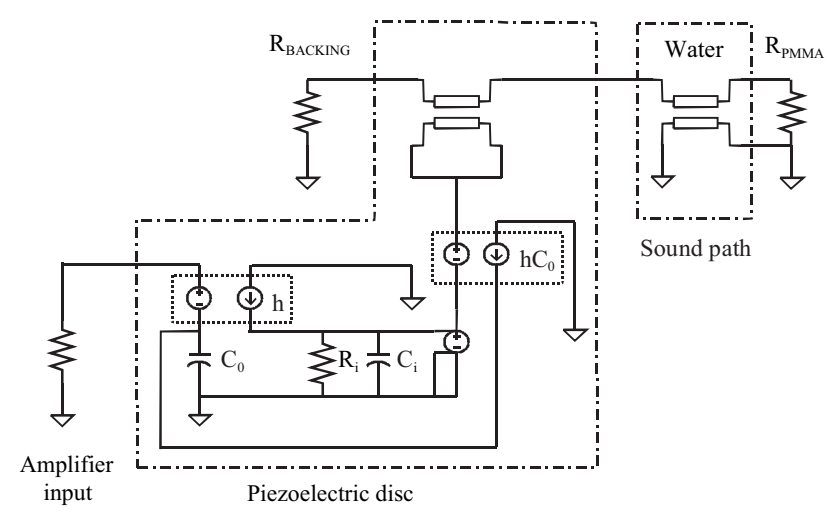

Fig. 4. Simulation setup with the piezoelectric disc in a pulse echo configuration. The medium is $5 \mathrm{~mm}$ of water, with pulse reflection in PMMA.

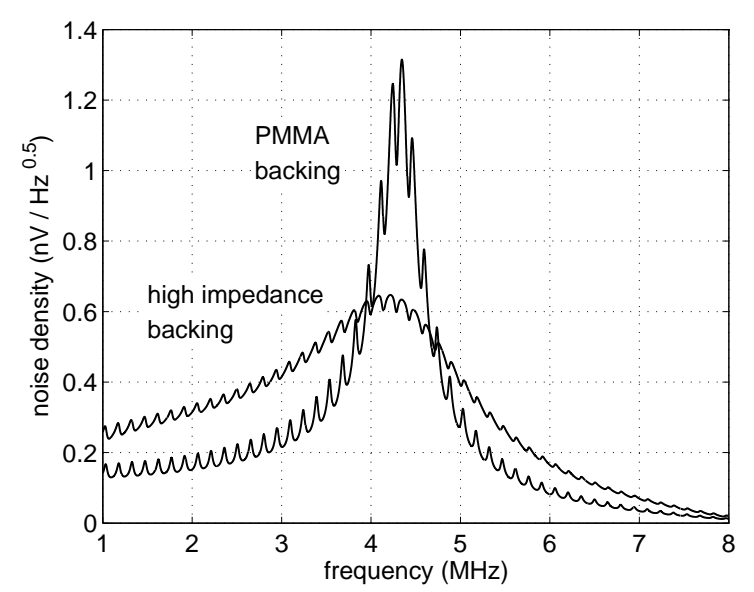

Fig. 5. Noise spectral density at transducer output when set up for pulse echo in water. The two curves are for PMMA backing, and for backing with acoustic impedance of five times PMMA.

the effect of the short distance of water, creating closely spaced resonances in the noise shaping curve.

\section{E Conclusions}

The inclusion of mechanical noise in a SPICE model of an ultrasound system has been performed by using the equivalence between thermal mechanical and thermal electrical noise. The results show a distinct shaping of the resulting noise spectral density, with a peak at the transducer resonance frequency. At the peak, the noise density reaches values in the range of low noise amplifiers which shows the relevance of the inclusion. The possibility to model the mechanical noise directly in the tool used for electronic and IC design will allow for efficient system level optimization, and avoid sub-optimization of the amplifier alone.

\section{F Literature}

[1] W. P. Mason, Electromechanical Transducers and Wave Filters. New York: Van Nostrand, 1942.
[2] M. Redwood, "Transient performance of a piezoelectric transducer," J. Acoust. Soc. Amer., vol. 33, pp. 527-536, April 1961.

[3] R. Krimholtz, D. A. Leedom, and G. L. Matthei, "New equivalent circuits for elementary piezoelectric transducers," Electronics Letters, vol. 6, pp. 398-399, 1970.

[4] W. M. Leach, "Controlled-source analogous circuits and SPICE models for piezoelectric transducers," IEEE Trans. Ultrason., Ferroelect., Freq. Contr., vol. 41, pp. 60-66, Januari 1994.

[5] A. Püttmer, P. Hauptmann, R. Lucklum, O. Krause, and B. Henning, "SPICE model for lossy piezoceramic transducers," IEEE Trans. Ultrason., Ferroelect., Freq. Contr., vol. 44, pp. 60-66, January 1997.

[6] J. van Deventer, T. Löfqvist, and J. Delsing, "PSpice simulation of ultrasonic systems," IEEE Trans. Ultrason., Ferroelec., and Freq. Contr., vol. 47, pp. 1014-1024, July 2000.

[7] J. Johansson, P.-E. Martinsson, and J. Delsing, "Simulation of absolute amplitudes of ultrasound signals using equivalent circuits," to appear in IEEE Trans. Ultrason., Ferroelec., and Freq. Contr., 2007.

[8] P. Tuinenga, SPICE: A Guide to Circuit Simulation and Analysis using PSpice. Englewood Cliffs, N.J.: PrenticeHall, 1995.

[9] R. Farlow and G. Hayward, "The minimum signal force detectable in air with a piezoelectric plate transducer," Proc. of the Royal Society of London, Series A, vol. 457, pp. 27412755, Nov. 2001.

[10] C. G. Oakley, "Calculation of ultrasonic transducer signalto-noise ratios using the klm model," IEEE Trans. Ultrason., Ferroelec., and Freq. Contr., vol. 44, pp. 1018-1026, September 1997.

[11] D. K. Cheng, Field and Wave Electromagnetics. AddisonWesley, 1989.

[12] J. B. Johnson, "Thermal agitation of electricity in conductors," Physical Review, vol. 32, pp. 97-109, July 1928.

[13] H. Nyquist, "Thermal agitation of electric charge in conductors," Physical Review, vol. 32, pp. 110-113, July 1928.

[14] H. B. Callen and T. A. Welton, "Irreversibility and generalized noise,” Physical Review, vol. 83, pp. 34-40, July 1951.

[15] R. Farlow and G. Hayward, "The absolute sensitivity of a piezocomposite transducer," in Proc. IEEE Int. Ultrason. Symp., vol. 2, pp. 883-886, 2001. 